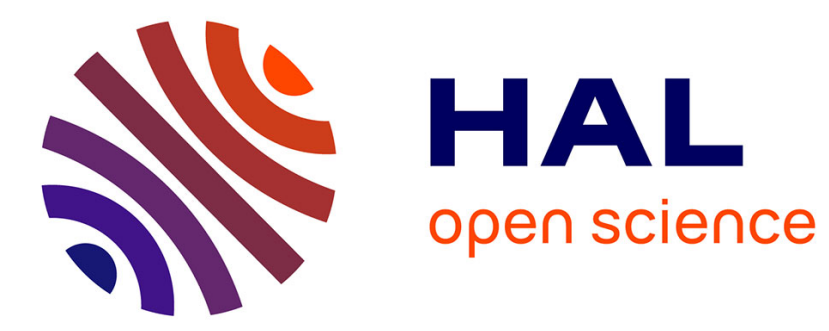

\title{
Perception of haptic motion is enhanced during conditions of increased postural stability
}

\author{
Fabien Vérité, S. Soria, R. Reynolds, Wael Bachta
}

\section{To cite this version:}

Fabien Vérité, S. Soria, R. Reynolds, Wael Bachta. Perception of haptic motion is enhanced during conditions of increased postural stability. Gait \& Posture, 2020, 76, pp.334-338. 10.1016/j.gaitpost.2019.12.023 . hal-02492120

\section{HAL Id: hal-02492120 \\ https://hal.sorbonne-universite.fr/hal-02492120}

Submitted on 26 Feb 2020

HAL is a multi-disciplinary open access archive for the deposit and dissemination of scientific research documents, whether they are published or not. The documents may come from teaching and research institutions in France or abroad, or from public or private research centers.
L'archive ouverte pluridisciplinaire $\mathbf{H A L}$, est destinée au dépôt et à la diffusion de documents scientifiques de niveau recherche, publiés ou non, émanant des établissements d'enseignement et de recherche français ou étrangers, des laboratoires publics ou privés. 


\title{
Perception of haptic motion is enhanced during conditions of increased postural stability
}

\author{
F. Vérité ${ }^{a, *}$, S. Soria ${ }^{a}$, R. Reynolds ${ }^{b}$ and W. Bachta ${ }^{a}$ \\ ${ }^{a}$ Sorbonne Université. Paris, France, CNRS, UMR 7222, Institut des Systèmes Intelligents et de Robotique, and INSERM, U1150, Agathe-Institut \\ des Systèmes Intelligents et de Robotique \\ ${ }^{b}$ School of Sport, Exercise and Rehabilitation Sciences, University of Birmingham, United Kingdom
}

\section{ARTICLE INFO}

\section{Keywords:}

Postural Balance

Light Touch

Sensory Integration

\begin{abstract}
A B S T R A C T
Background: Coupling between postural sway and fingertip displacement has been observed in individuals lightly touching a moving surface. This can be attributed to the central nervous system (CNS) misinterpreting surface motion as self-motion, evoking a compensatory sway response.

Research question: Does baseline postural state influence the correct perception of haptic object motion?

Methods: Motion perception detection thresholds of index finger displacement at $1 \mathrm{~mm} . \mathrm{s}^{-1}$ velocity during light touch were determined for three postural conditions: standing with eyes open (EO) and closed (EC), and sitting with eyes closed. For the standing condition with eyes shut, displacement thresholds were measured using three velocities $\left(1,2 \& 4 \mathrm{~mm} . \mathrm{s}^{-1}\right)$.

Results: Postural condition had a large influence on motion perception, with a reduction in displacement threshold from $12 \rightarrow 6 \rightarrow 2 \mathrm{~mm}$ during the transition from standing $\mathrm{EC} \rightarrow$ standing $\mathrm{EO} \rightarrow$ sitting EC. A systematic decrease in displacement perception threshold was observed with increasing velocity. This tends to suggest that the increase of the touched object velocity may help overcoming the misinterpretation.

Significance: These results suggest that the ability to disambiguate self motion from haptic motion is enhanced during stable postures, and when stimulus velocity is high. Our findings may help to understand the mechanisms underlying the coupling between surface movements and postural sway, reported in the literature.
\end{abstract}

\section{Introduction}

Postural control relies on the integration of a number of sensory inputs including vestibular, visual, proprioceptive and haptic information. Haptics include both proprioception and tactile feedback. With respect to haptic input, balance-relevant feedback arises from the plantar surface of the foot [1]. It also arises from the hand when making manual contact with an earth-fixed object [2], or another person [3]. Such contact is as equally effective as vision for enhancing postural control, with up to $50 \%$ reductions in sway reported when touching a fixed object $[4,5]$. The benefits of touch are observed even when contact forces are too low to offer mechanical support [5], and are abolished if the arm is anaesthetized [6]. This implies that light touch information from the fingers is integrated with other sensory modalities for postural control.

Although touching a stationary surface improves balance, contact with a moving surface actually evokes postural sway $[7,8]$. Body movement is observed at the same frequency as the stimulus, with peak response gain seen around 0.2-0.4 Hz, diminishing at higher frequencies. We have recently utilized the haptic-evoked sway response in order to control body position of a person touching a robotically-controlled moving surface driven by real-time feedback of body sway [9]. The evoked sway suggests that object motion is misinterpreted as self-motion, and that the observed response is designed to compensate for a false sensation of body sway. This is analogous to visually-evoked sway, whereby a slowly-moving scene produces an illusory sense of self-motion ( $a k a$ vection) accompanied by a compensatory body sway response [10].

If haptic-evoked sway is caused by object-motion being misinterpreted as self-motion, anything which alerts the subject to object-motion may suppress the sway response. For example, there is evidence that sway responses to haptic

\footnotetext{
*Corresponding author verite@isir.upmc.fr ORCID(s):
} 
Perception of haptic motion is enhanced during conditions of increased postural stability

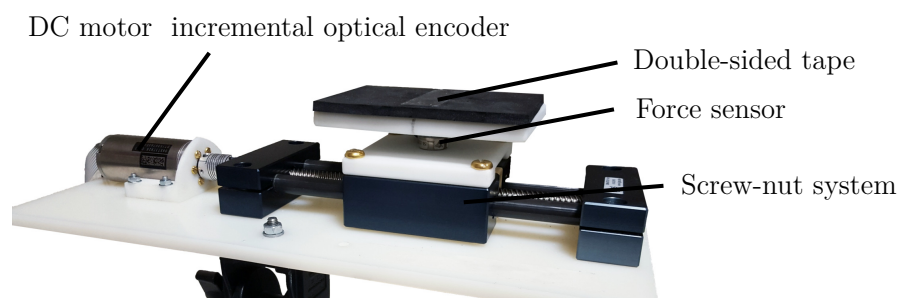

Figure 1: The experimental device is composed of a moving surface powered by a DC motor and its associated encoder. The latter enables the measurement and the control of the surface position.

stimuli habituate on successive trials, once the subject becomes aware of the destabilizing effect of their response [11]. Another factor which may influence the ability to distinguish object- from self-motion is baseline postural stability. For example, when posture is poorly controlled and body sway is high, it may be more difficult to disambiguate sensory exafference from reafference [12]. Conversely, when body sway is minimal, any external perturbations may be easier to detect. Thus, the propensity to succumb to such a perturbation may depend upon one's baseline postural state. Here, we ask if the ability to detect haptic motion at the fingertip is influenced by postural state. Specifically, we determine the threshold of finger motion perception during light touch under three postural conditions; Standing with eyes closed or open, and sitting with eyes closed. We hypothesize that object motion will be easier to detect under conditions of increasing postural stability. Moreover, we predict that increasing the velocity of object motion will also help this disambiguation, when standing.

\section{Methods}

\subsection{Participants}

We recruited nine subjects (5 females; mean age of 26.2 years (Standard Deviation (SD) of 1.85)), with no known neurological abnormalities. The study was carried out in conformity with the Declaration of Helsinki of the World Medical Association, and all participants gave their informed consent. The study received the approval of the local scientific and ethics committee (CERES).

\subsection{Experimental setup}

The experimental setup is depicted in Figure 1. The surface which subjects made finger contact consists of a plastic plate. This is covered in double-sided sticky tape to help maintain contact of the finger with the surface. The device moves the participant's finger evoking both proprioceptive and cutaneous sensory information. The cutaneous information arises from the digit pulp elasticity. The plate is translated by a nut-screw driven by a DC servo motor equipped with an optical encoder of 0.0015 rad resolution, equating to a linear resolution of $0.003 \mathrm{~mm}$ when taking into account the gear ratio of the lead screw mechanism. During the experiment, the lead screw was placed in front of participants and oriented to produce translation in the sagittal plane (i.e. forward/backward motion) as it was done by Wing et al [8]. The motor is controlled by an Elmo Solo-Whistle servo drive. An ATI Nano 17 force sensor measured forces normal to the plastic plate. An audible alarm was triggered if this force exceeded $1 \mathrm{~N}$. The motor was controlled by custom-written $\mathrm{C}$ software running under Xenomai real time system at a refresh rate of $500 \mathrm{~Hz}$. Pink noise was broadcast in the experimental room to remove any auditory cues from the motor.

\subsection{Experimental procedure}

The experiment was designed to assess perceptual thresholds of surface displacement when subjects made contact with the moving plate using their right index finger, under different postural conditions and surface velocities. The objective is to evaluate the amplitude of the finger movement necessary to the detection of its direction. During our experiment, we tried not to favorize any sensory information. Indeed, participants kept the same upper arm configuration during the different trials. The upper arm joints were set far from the joints limits.

The upper-limb postural configuration has been controlled visually to be the same for each participant during the sitting and the standing positions. Moreover, we paid attention to let the arm configuration away from the joints limits, where proprioception can be biased [13].

Various methods to determine displacement detection thresholds exist [14]. We employed a method similar to the one used by Hall \& McCloskey [15], but slightly modified to reduce experimental duration. Instead of using a large 


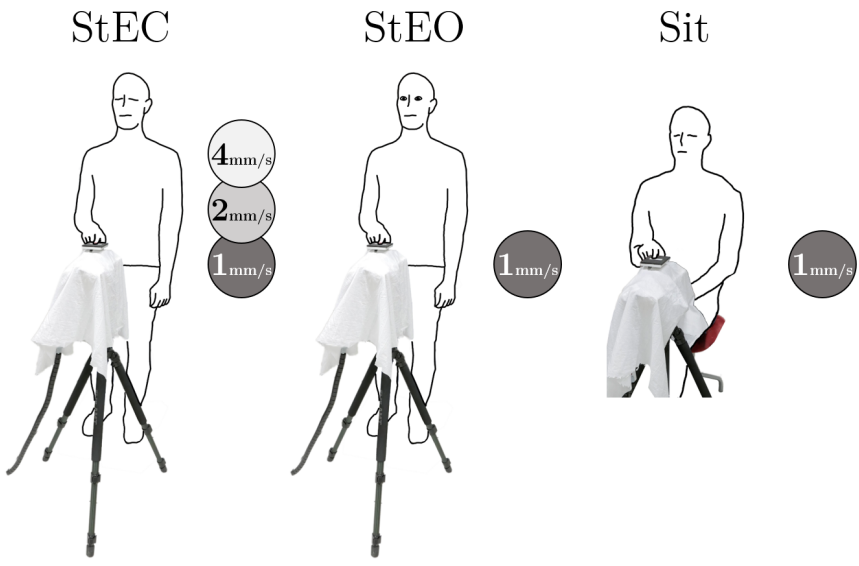

Figure 2: The five experimental conditions were divided in three postural conditions (Standing with Eyes Closed, Standing with Eyes Open and Sitting with Eyes Closed) and three finger displacement velocities (1, 2 or 4 mm. $\left.\mathrm{s}^{-1}\right)$. The 5 conditions are StECx1, StECx2, StECx4, StEO and Sit.

number of different velocities, we have focused on only three. Moreover, for each velocity, Hall \& McCloskey used incremental steps in order to determine the threshold. Here, we have chosen to use a staircase-like method. This was necessary to avoid fatigue, since participants were standing most of the time.

Five conditions were tested. For each condition, displacement detection threshold was repeated over three trials. All trials were presented randomly. Each trial was composed of a different number of runs. For each run, participants were asked to report the direction (forward or backward) of 10 successive platform movements (i.e. their own index finger motion). They were constantly reminded during the experimentation to be sure of their answer, and not to guess. The experimental protocol was divided into three sessions over separate days in order to avoid fatigue and keep participants focused throughout. Each participant finished the protocol within a week, during three 60 minutes sessions.Since trials are randomized, one session does not necessarily include one trial of each condition.

\subsubsection{Experimental conditions}

The three postural conditions were as follows: sitting with eyes closed (Sit), standing with eyes open (StEO), and standing with eyes closed (StEC). For the Sit and StEO conditions, platform displacement velocity was set to $1 \mathrm{~mm} . \mathrm{s}^{-1}$. For the StEC condition, two additional velocities were included, specifically $2 \& 4 \mathrm{~mm} . \mathrm{s}^{-1}$. This produced a total of 5 conditions: StEO, Sit, StECx1, StECx2, and StECx4 (the three last names correspond the StEC with the 1, 2 and $4 \mathrm{~mm} . \mathrm{s}^{-1}$ platform displacement velocity, see Fig. 2). In the standing position, the translational device was placed at the same height as the participant's center of mass. In the sitting position, the upper limb configuration was controlled as described above.

\subsubsection{Determining threshold of motion detection}

During each trial, participants were asked to lightly touch the mobile surface. The double-sided tape insured a permanent contact between their finger and the moving surface. They were aware that the surface was going to move. During the trial, a given number of runs were presented; each run is composed of 10 successive surface movements. At the end of each movement, participants had 5 seconds to verbally report whether movement direction was forward or backward, or if they did not know. At the end of the run their answers were processed to determine a score, which was used to compute the displacement magnitude for the following run.

The movements are presented randomly but the platform comes back to its original position after the 10 movements. The maximal observed displacement amplitude was $4.5 \mathrm{~cm}$ during any trial. Depending on the amplitude and velocity, a run duration varies approximately between $40 \mathrm{~s}$ and $3 \mathrm{~min}$. The scores were calculated as follows:

- +1 : if correct.

- 0: if the participant did not know. 
Perception of haptic motion is enhanced during conditions of increased postural stability
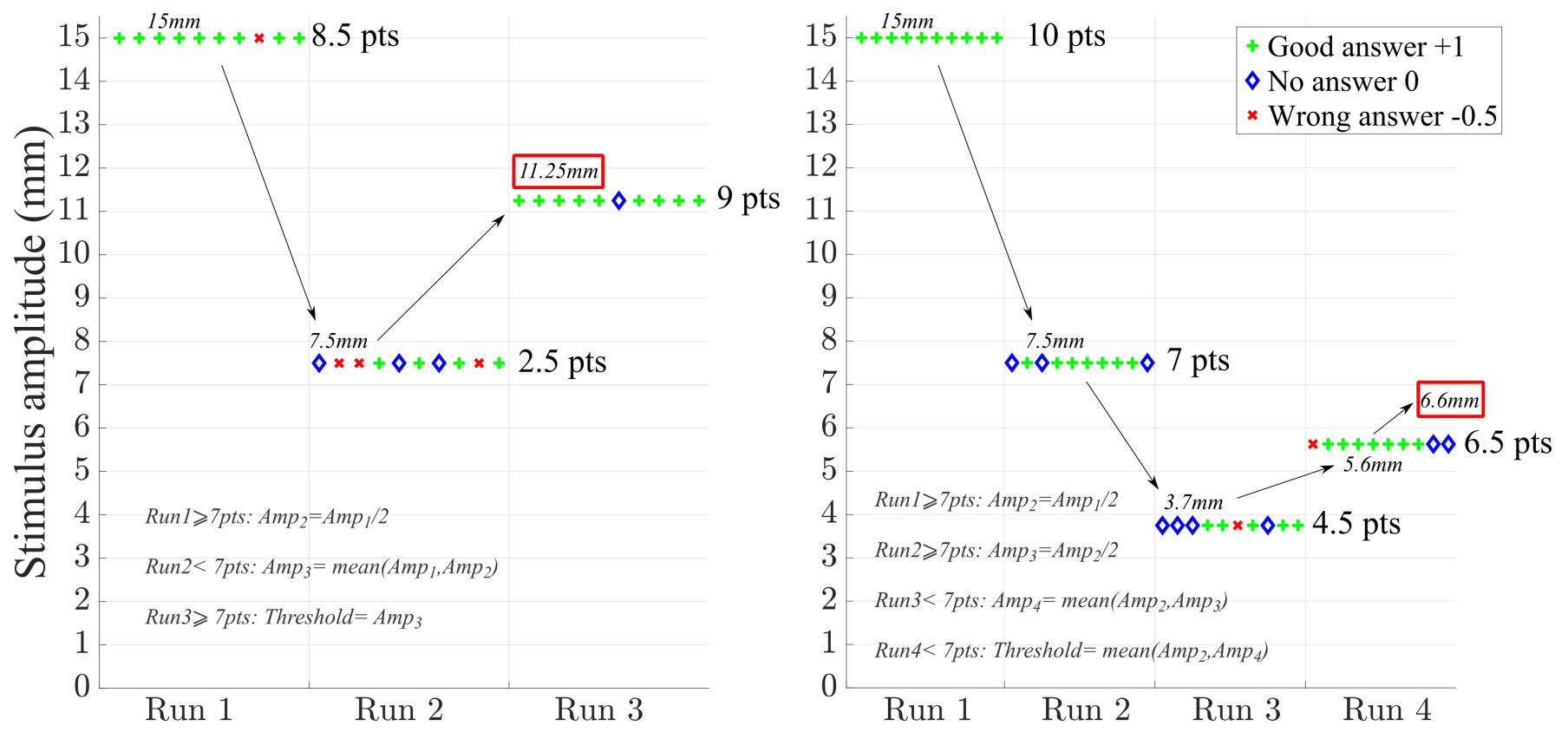

Figure 3: Threshold measurement for two trials for one participant. (Left) Here it can be observed that it takes three runs to find the threshold. In the first run $(15 \mathrm{~mm})$, the subject managed to detect the direction of the stimulus. Therefore the stimulus amplitude is divided by 2 in the second run. In the second run, the participant did not succeed. Finally, the amplitude of the stimulus was raised (as the average of the two last ones). The participant managed to detect the direction for this last stimulus, this final amplitude is accounted as the detection threshold for this participant in this condition. / (Right) Here it can be observed that it takes four runs to find the threshold. In the first two runs (15mm and $7.5 \mathrm{~mm}$ ), the subject managed to detect the direction of the stimulus. In the third run $(3.7 \mathrm{~mm})$, the participant did not succeed. Finally, the amplitude of the stimulus was raised (as the average of the two last ones). The participant did not manage to detect the direction for this last stimulus, the threshold is thus calculated as the mean between the last amplitude that the participant had been able to detect and the last one.

\section{- -0.5: if incorrect.}

A displacement detection was considered successful if the score was equal to, or higher than, 7 (from a possible maximum of 10). If the run was successful, displacement amplitude was halved for the following run. If unsuccessful, displacement was increased to the mean value of the last successful and unsuccessful runs. The detection threshold was then determined by the result of the following run: If successful, the amplitude of this run taken as the threshold. If unsuccessful, the mean value of the amplitude of the last run and of the last successfully detected run was taken as the threshold. The first tested motion magnitude was set to $1.5 \mathrm{~cm}$ for the standing positions and $1 \mathrm{~cm}$ for the sitting position. Figures 3 illustrates score computing for one participant.

\subsection{Statistical analyses}

For each condition, thresholds were determined from three trials. A repeated measure ANOVA gave no statistically significant differences between first, second and third trials over all participants and conditions. Therefore, the mean of the thresholds obtained during the three trials was considered to be the participant's displacement threshold in the considered condition i.e. the dependant variable for the following analyses.

The data from the five conditions have been used to carry out two separate inferential statistical procedures. The first one aims to show that increasing postural stability leads to low detection threshold. The three levels of the stability factor have been included i.e. Sit, StEO and StECx1.

The second analysis aimed to highlight the effect of increasing stimulus velocity, during standing. Three levels of velocity have been included: StECx1, StECx2 and StECx4. Only the StEC balance stability factor has been performed with different stimulus velocities. This factor has been chosen since it has been expected to display the worst motion 


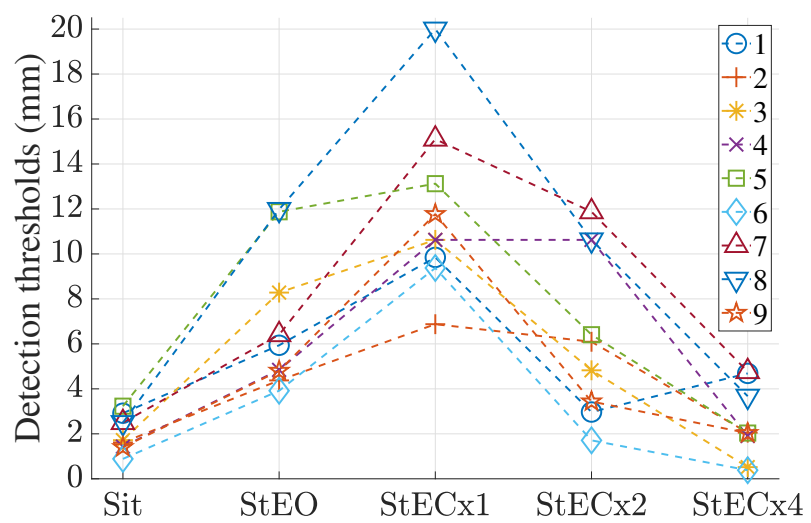

Figure 4: Displacement detection threshold for each individual across all conditions. The nine subjects are depicted with different colors and markers.

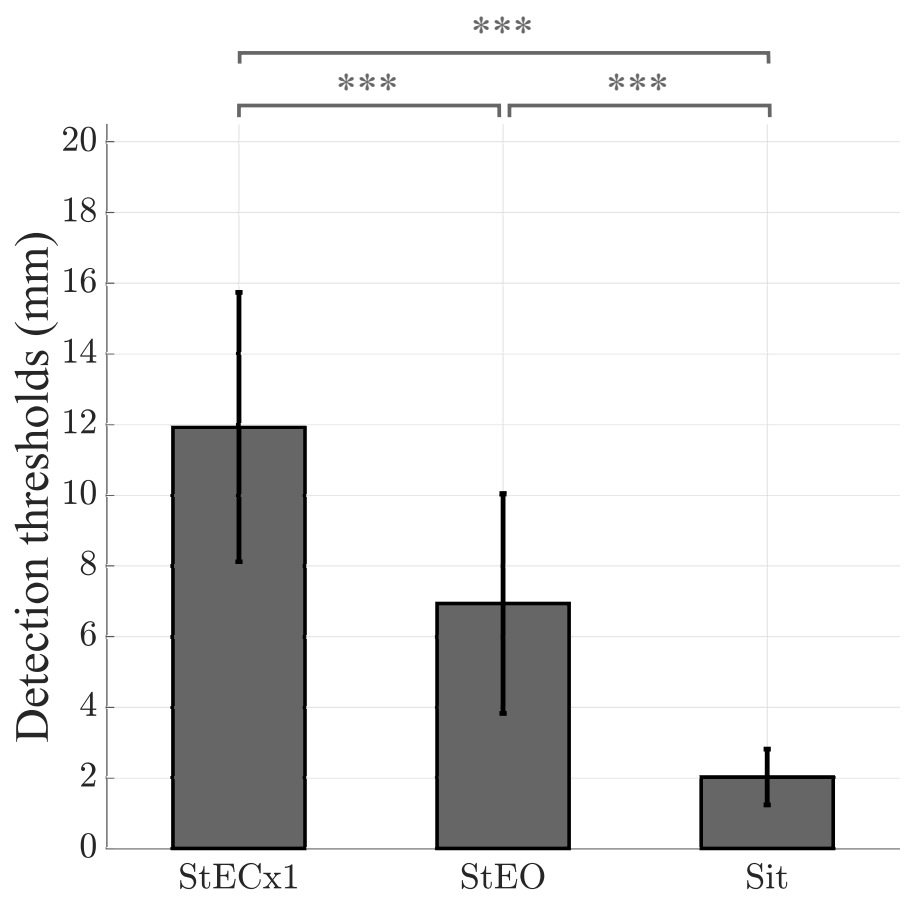

Figure 5: Displacement detection thresholds for the three postural conditions with a finger velocity of $1 \mathrm{~mm} . \mathrm{s}^{-1}$. Bar heights correspond to mean values and error bars correspond to standard deviations.

detection rate.

For each statistical procedure, The Shapiro Wilk and Maulchy's tests were used to check the normality and sphericity of collected data.

Finally, repeated-measures ANOVA was used to evaluate statistical differences among the conditions. Following a significant ANOVA result, the Tukey post hoc test was used to determine which conditions are different from each other. Statistical significance was set to $p<0.05$.

\section{Results}

Individual displacement detection thresholds can be seen in figure 4. For all nine subjects, the highest threshold occurred when standing with a surface movement velocity of $1 \mathrm{~mm} \cdot \mathrm{s}^{-1}$ (StECx1). Opening the eyes or sitting down both reduced the threshold, as did increasing surface velocity. The two ANOVA analyses confirmed our results. 


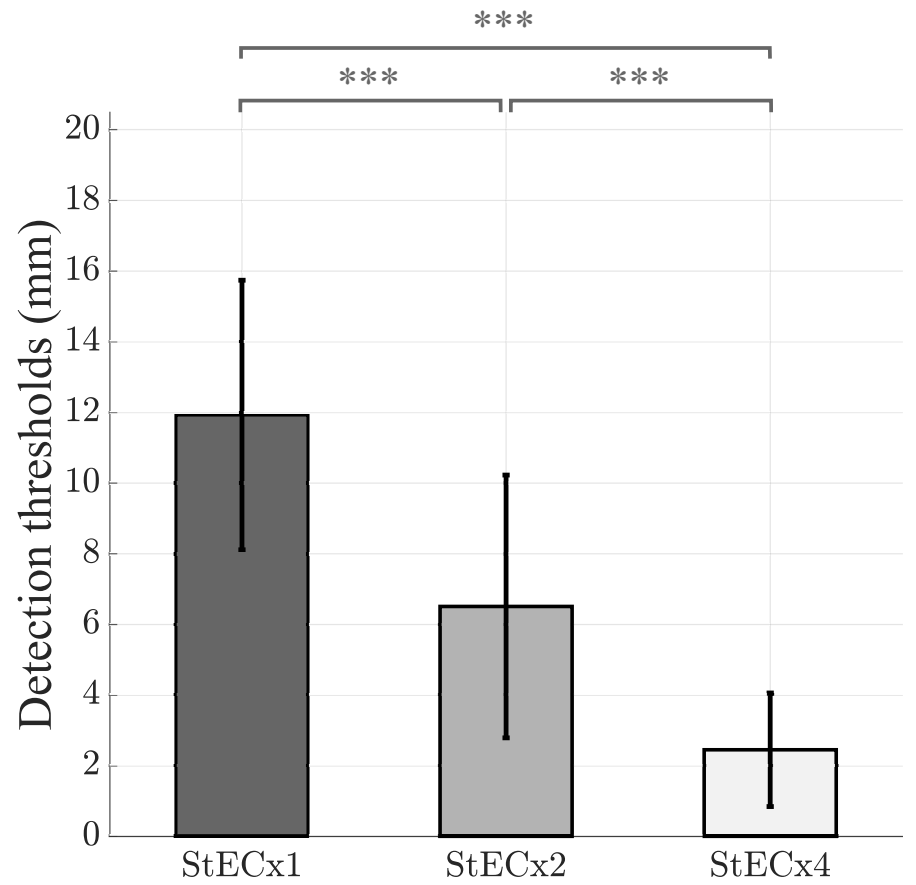

Figure 6: Displacement detection thresholds for the three different velocities in the upright posture with the eyes closed. Bar heights correspond to mean values and error bars correspond to standard deviations.

The first one showed a significant effect of the baseline postural stability upon detection threshold $\left(F_{(2,16)}=\right.$ $50.2 ; p<0.0001)$. The effect of baseline postural condition is shown in figure 5 , which depicts mean data for all the conditions with a surface velocity of $1 \mathrm{~mm} . \mathrm{s}^{-1}$. Post-hoc analysis confirms a reduction in threshold from standing with eyes closed (StECx1) to standing with eyes open (StEO), and a further reduction during sitting (Sit; $p<0.00001$ ). The second one showed a significant effect of the stimulus velocity upon detection threshold $\left(F_{(2,16)}=36.2 ; p<\right.$ $0.0001)$. The effect of surface velocity during the standing condition is shown in figure 6 . Increasing velocity caused a systematic reduction in detection threshold from $\sim 12$ to 6 to $2 \mathrm{~mm}$ (StECx1 to StECx2 to StECx4; $p<0.001$ ).

\section{Discussion}

Our main finding is that the ability to detect surface motion when making light finger contact with a horizontal surface is systematically altered by postural state. During the transition from sitting to standing with eyes open, and then to standing with eyes closed, it became increasingly difficult to detect surface motion. This manifested as an increase in the amplitude of movement necessary to achieve perceptual detection. In addition to the effect of postural condition, there was also a systematic reduction in detection threshold as movement velocity increased. Firstly, we discuss the potential mechanisms underlying the effect of postural state, and then we consider the effect of movement velocity.

\subsection{The influence of postural state upon movement detection.}

It is well established that, when touching a fixed surface, haptic input from the hand can be used to stabilize posture when standing [4]. This benefit of touch is seen even when interaction forces are too low to provide mechanical stabilization, emphasizing the role of haptic feedback for balance [6]. However, if the touched surface is made to move, postural sway is actually evoked. In this case, surface movement must be sufficiently slow to mimic the pattern of forces at the fingertip during naturally-occurring sway. This causes the haptic feedback to be misinterpreted as self-motion by the central nervous system (CNS). This is equivalent to visually-evoked vection, whereby slow movement of the visual scenery evokes a counteractive sway response in the same direction as scene motion [16]. Similarly, motion of the haptic surface is misinterpreted as postural sway in the direction opposite stimulus motion, and a counteractive response is observed in the same direction as the stimulus. In both cases, the CNS is unable to fully distinguish between sensory feedback caused by self motion (reafference) from that caused by external motion (exafference). 
In the current study we investigated this ambiguity from a different perspective. Rather than measuring evoked sway responses to moving haptic stimuli, we determined the ability to perceive such motion. Nevertheless, the task presented to the CNS is equivalent i.e. to differentiate between reafference and exafference. We observed that the perceptual ability to detect haptic motion was profoundly affected by postural state. Perceptual performance was best when seated, and worst when standing with eyes closed. In fact, perceptual threshold amplitudes differed by a factor of six between these two extremes $(12 \mathrm{~mm} v s 2 \mathrm{~mm})$. This suggests that, in conditions where baseline postural sway is minimized, perception of external surface motion is greatly enhanced. In other words, any ambiguity between self motion and external motion is much reduced. This presumably occurs because, as baseline sway diminishes, the magnitude of haptic feedback evoked by the moving surface becomes greater than that caused by naturally-occurring sway. Hence, it is more easily distinguished from continuous 'background' reafference. This explanation is consistent with the findings of Misiaszek et al [11, 17], who showed that haptic-evoked sway responses were larger under conditions of elevated baseline sway. Nevertheless, the fact that such ambiguity occurs at all does highlight the fact that the CNS struggles to predict the pattern of reafference produced by naturally - occurring sway. Although we measured perception, rather than sway, previous research on the visual system suggests that similar mechanisms are involved in distinguishing between self- and external motion for both perception and action [18]. Futhermore, visually-evoked sway responses correlate with the magnitude of simultaneously reported vection sensations [10]. However, perception may be more amenable to modulation by cognitive factors than sway. For example, Lackner \& DiZio [19] reported that subjects sometimes perceived a stationary haptic stimulus as moving, but only if they were told in advance that it might move. Similarly, during the pre-test session we observed that some subjects reported surface movement in conditions where the surface was kept stationary. This suggests that prior expectation can bias the perception of haptic stimuli. Whether this translates to the motor system is unknown. Such cognitive effects have previously been reported for visually-evoked sway responses [20,21]; but not for proprioceptive- or vestibular-evoked responses [22, 23]. The observation that haptic-evoked sway responses rapidly habituate would suggest that they are amenable to cognitive factors [11]. Furthermore, Jeka et al [24] suggested that conscious perception of a haptic stimulus may affect its ability to evoke sway. They reported a weaker coupling between touch bar motion and sway in a participant who consciously perceived the motion, compared with those who did not. The authors suggest that "Cognitive mechanisms might introduce flexibility into the postural control scheme and allow for parameters that may be fixed at the reflexive level to be adaptively changed" [24]. Nevertheless, the precise relationship between haptic motion perception and the concomitant sway response is unknown.

Bryanton et al [25] studied the influence of light touch on balance when the touch surface becomes unreliable. They found that sway area is always decreased when compared to a condition without touch (eyes closed), even after three successive movements of the touched surface. They explain their results as an under-weighting of the somatosensory signal, or a minimization of sway in order to enhance perception of finger movement. The last hypothesis is compatible with our results. However, one should keep in mind that the maximal stimulus velocity that we used is the third of the one used by Bryanton et al.

\subsection{The influence of stimulus velocity upon movement detection.}

During the standing/eyes closed condition we systematically altered stimulus velocity. Increasing the stimulus from 1 to $2 \mathrm{~mm} . \mathrm{s}^{-1}$ almost halved the perceptual threshold, and a further increase to $4 \mathrm{~mm} . \mathrm{s}^{-1}$ caused a significant further drop. Hence, increasing displacement velocity helped to overcome the stimulus ambiguity caused by standing. In the sitting position, the sway is assumed to be negligible.

Postural adjustments and cognitive processes associated with repetitive stimuli could arise. Here, the sway was not measured but one can suppose that the postural corrections would be bigger for higher velocity stimuli. Hence the sway pattern should increase from 1 to 2 to $4 \mathrm{~mm} / \mathrm{s}$. If true, this would only serve to dilute the observed effect of stimulus velocity upon perceptual detection. Therefore, any postural sway directly evoked by the stimulus cannot explain our results.

This substantial change in perceptual performance can be explained by considering the physiology of the sensory receptors in combination with the nature of postural sway when standing. Firstly, somatosensory receptors are inherently very sensitive to velocity [26]. Hall \& McCloskey [15] studied perceptual thresholds for joint movements and reported a consistent reduction in threshold with increasing joint velocity. Directly relevant to our findings, they also reported that the minimum threshold for the perception of finger translation was $\sim 2 \mathrm{~mm}$ for a velocity of $1 \mathrm{~mm} \cdot \mathrm{s}^{-1}$. This is very similar to the values we report here for the sitting condition. When they increased stimulus velocity to $4 \mathrm{~mm} . \mathrm{s}^{-1}$, the threshold reduced to $\sim 0.5 \mathrm{~mm}$. When we compare our reported thresholds to the extent of body motion during 
standing, it compares favourably. Jeka [27] reported the anteroposterior centre of mass velocity to be $1.7 \pm 3.5 \mathrm{~mm} . \mathrm{s}^{-1}$ during normal standing with eyes closed. A haptic stimulus velocity of $1 \mathrm{~mm} \cdot \mathrm{s}^{-1}$ is therefore very close to the typical velocity of body motion during standing, making it difficult to distinguish from postural sway. Increasing this to $4 \mathrm{~mm} \cdot \mathrm{s}^{-1}$ could allow it to be more easily distinguished from sway motion.

\section{Conclusion}

Previous research has demonstrated that haptic contact with a moving surface can evoke postural sway when standing. Here we show that perception of haptic motion is strongly dependent upon the nature of the postural condition. As posture became increasingly more stable, the ability to detect a moving haptic stimulus was greatly enhanced. Hence, the ability to distinguish exafferent from reafferent haptic information depends upon baseline postural state, and also upon the kinematic properties of the exafferent stimulus.

\section{References}

[1] a. Kavounoudias, R. Roll, J. P. Roll, Foot sole and ankle muscle inputs contribute jointly to human erect posture regulation, Journal of Physiology 532 (3) (2001) 869-878. doi:10.1111/j.1469-7793.2001.0869e.x.

[2] L. Assländer, C. P. Smith, R. F. Reynolds, Sensory integration of a light touch reference in human standing balance, PLoS ONE 13 (6) (2018) 1-20. doi:10.1371/journal.pone.0197316.

[3] R. F. Reynolds, C. J. Osler, Mechanisms of interpersonal sway synchrony and stability, Journal of the Royal Society Interface 11 (101) (2014). doi:10.1098/rsif.2014.0751.

[4] J. J. Jeka, J. R. Lackner, Fingertip contact influences human postural control., Experimental brain research 100 (3) (1994) 495-502.

[5] M. Holden, J. Ventura, J. R. Lackner, Stabilization of posture by precision contact of the index finger, Journal of vestibular research : equilibrium \& orientation 4 (4) (1994) 285-301.

[6] M. Kouzaki, K. Masani, Reduced postural sway during quiet standing by light touch is due to finger tactile feedback but not mechanical support., Experimental brain research 188 (1) (2008) 153-8. doi:10.1007/s00221-008-1426-5.

[7] J. Jeka, K. Oie, G. Schöner, T. Dijkstra, E. Henson, Position and velocity coupling of postural sway to somatosensory drive., Tech. Rep. 4, Department of Kinesiology, University of Maryland, College Park, Maryland 20742, USA. (1998). doi : citeulike-article-id:524528.

[8] A. M. Wing, L. Johannsen, S. Endo, Light touch for balance: influence of a time-varying external driving signal., Philosophical transactions of the Royal Society of London. Series B, Biological sciences 366 (1581) (2011) 3133-41. doi : 10.1098/rstb . 2011.0169.

[9] F. Verite, W. Bachta, G. Morel, Closed Loop Kinesthetic Feedback for Postural Control Rehabilitation, IEEE Transactions on Haptics 7 (2) (2014) 150-160. doi:10.1109/T0H.2013.64.

[10] A. E. Thurrell, A. M. Bronstein, Vection increases the magnitude and accuracy of visually evoked postural responses, Experimental Brain Research 147 (4) (2002) 558-560. doi:10.1007/s00221-002-1296-1.

[11] J. Misiaszek, J. Forero, E. Hiob, T. Urbanczyk, Automatic postural responses following rapid displacement of a light touch contact during standing, Neuroscience 316 (2016) 1-12. doi:10.1016/j . neuroscience.2015.12.033.

[12] K. E. Cullen, Sensory signals during active versus passive movement, Current Opinion in Neurobiology 14 (6) (2004) 698-706. doi: $10.1016 / j$. conb. 2004.10.002.

[13] U. Proske, S. C. Gandevia, The Proprioceptive Senses: Their Roles in Signaling Body Shape, Body Position and Movement, and Muscle Force, Physiological Reviews 92 (2012) 1651-1697. doi:10.1152/physrev.00048.2011.

[14] M. R. Leek, Adaptive procedures in psychophysical research., Perception \& psychophysics 63 (8) (2001) 1279-92. doi:10.3758/ BF03194543.

[15] L. a. Hall, D. I. McCloskey, Detections of movements imposed on finger, elbow and shoulder joints., The Journal of physiology 335 (1983) $519-533$.

[16] D. Lee, J. Lishman, Visual proprioceptive control of stance., Journal of Human Movement Studies 1 (January) (1975) 87-95.

[17] J. E. Misiaszek, J. Vander Meulen, Balance reactions to light touch displacements when standing on foam, Neuroscience Letters 639 (2017) 13-17. doi:10.1016/j.neulet.2016.12.027.

[18] A. Reichenbach, J. Diedrichsen, Processing reafferent and exafferent visual information for action and perception, Journal of Vision 15 (8) (2015) 1-12. doi:10.1167/15.8.11.

[19] J. R. Lackner, P. A. DiZio, Aspects of body self-calibration, Trends in Cognitive Sciences 4 (7) (2000) 279-288. doi:10.1016/ S1364-6613(00)01493-5.

[20] A. M. Bronstein, Suppression of visually evoked postural responses, Experimental Brain Research 63 (3) (1986) 655-658. doi:10.1007/ BF00237488.

[21] M. Guerraz, K. V. Thilo, A. M. Bronstein, M. A. Gresty, Influence of action and expectation on visual control of posture, Cognitive Brain Research 11 (2) (2001) 259-266. doi:10.1016/S0926-6410(00) 00080-X.

[22] M. Guerraz, B. L. Day, Expectation and the vestibular control of balance., Journal of cognitive neuroscience 17 (3) (2005) 463-469. doi: 10.1162/0898929053279540.

[23] S. Caudron, F. Boy, N. Forestier, M. Guerraz, Influence of expectation on postural disturbance evoked by proprioceptive stimulation, Experimental Brain Research 184 (1) (2008) 53-59. doi :10.1007/s00221-007-1079-9.

[24] J. J. Jeka, G. Schöner, T. Dijkstra, P. Ribeiro, J. R. Lackner, Coupling of fingertip somatosensory information to head and body sway., Experimental brain research 113 (3) (1997) 475-83. 
Perception of haptic motion is enhanced during conditions of increased postural stability

[25] M. A. Bryanton, S. D. C. Chodan, J. Vander Meulen, K. K. Fenrich, J. E. Misiaszek, The effect of light touch on standing sway when the stability of the external touch reference becomes unreliable, Experimental Brain Research 237 (3) (2019) 663-672. doi:10.1007/ s00221-018-5455-4.

[26] P. B. C. Matthews, Proprioceptors and their contribution to somatosensory mapping; complex messages require complex processing, Canadian Journal of Physiology and Pharmacology 66 (4) (1988) 430-438. doi : 10.1139/y88-073.

[27] J. Jeka, T. Kiemel, R. Creath, F. Horak, R. Peterka, Controlling human upright posture: velocity information is more accurate than position or acceleration., Journal of neurophysiology 92 (4) (2004) 2368-79. doi:10.1152/jn.00983.2003. 